\title{
DeKaRT: A New Paradigm for Key-Dependent Reversible Circuits
}

\author{
Jovan D. Golić \\ System on Chip, Telecom Italia Lab \\ Telecom Italia \\ Via Guglielmo Reiss Romoli 274, I-00148 Turin, Italy \\ jovan.golic@tilab.com
}

\begin{abstract}
A new general method for designing key-dependent reversible circuits is proposed and concrete examples are included. The method is suitable for data scrambling of internal links and memories on smart card chips in order to foil the probing attacks. It also presents a new paradigm for designing block ciphers suitable for small-size and/or high-speed hardware implementations. In particular, a concrete building block for such block ciphers with a masking countermeasure against power analysis incorporated on the logical gate level is provided.
\end{abstract}

Keywords. Keyed reversible circuits, data scrambling, block ciphers, countermeasures, probing attacks, power analysis.

\section{Introduction}

Probing attacks on microelectronic data-processing devices implementing cryptographic functions, such as smart cards, are invasive techniques consisting in introducing conductor microprobes into certain points of a tamper-resistant chip within the device to monitor and analyze the electrical signals at these points, in order to recover some information about the secret key used (see [1]). They can be classified as side-channel attacks if they do not change the functionality of the device. In this regard, potentially vulnerable points are those corresponding to internal links or memories that are likely to convey or contain secret information and whose hardware implementation has a regular, recognizable structure. In a microprocessor configuration, the RAM and the bus connecting it to the microprocessor are specially vulnerable, and the bus between the microprocessor and cryptoprocessor(s) may also be vulnerable. While, for a cryptographic function, it should be computationally infeasible to reconstruct the secret key from known input and output data, this need not be the case if intermediate data generated during the software execution is revealed. Therefore, there is a need to protect the sensitive data on data buses and in memories by using dedicated encryption techniques. Apart from data, one can also encrypt the memory addresses and the code instructions. This encryption may also reduce the vulnerability to other side-channel attacks such as the power analysis attacks. 
The encryption/decryption of data solely on the data bus can be achieved by a fast stream cipher combining the data sequence with the keystream sequence, produced by a centralized random or pseudorandom number generator, possibly by the bitwise XOR operation. However, this solution is not satisfactory for encrypting the data to be stored in memories as the same keystream block has to be used for encrypting and decrypting any data block for a particular memory location. The keystream block can be made dependent on the location itself, but is immediately recovered from only one known pair of original and encrypted data blocks. A more satisfactory solution for encrypting the memory data, which can also be used for encrypting the bus data, is to apply a block cipher, in the electronic code book mode, and the encryption has to be performed by logical circuits typically in a single microprocessor clock cycle, as it is then transparent to the remaining components of the data-processing device. However, the usage of classical block ciphers such as DES 13] or AES [5] is not realistic due to very restrictive high-speed and small-size requirements and, also, because of small and variable block sizes involved.

Accordingly, there is a need for fast and simple techniques for key-controlled reversible transformations which, of course, cannot achieve the same security level as classical block ciphers and are therefore called data scrambling instead of encryption. Nevertheless, it has to be noted that the probing attacks are not easy to mount and consequently partial instead of full knowledge of ciphertext corresponding to known, possibly chosen, plaintext is a much more appropriate assumption for data scrambling. To this end, one can use simplified iterated designs for block ciphers, with a reduced block size and with a reduced number of rounds, but the usual method of bitwise XORing the expanded secret key with intermediate ciphertexts is not good enough for relatively small block sizes. Instead, one can incorporate a larger number of key bits by using key-controlled bit permutations to permute bits in a block. Several constructions for such permutations are proposed in [3] for block sizes being a power of 2. Nevertheless, a small number of rounds and the linearity of bit permutations do not allow one to achieve a sufficient security level especially if the block size is very small.

The main objective of this paper is to introduce a new and generic method for iterated construction of key-controlled reversible transformations which can incorporate a large number of key bits in a small number of rounds even for very small block sizes. The new, so-called DeKaRT method is based on using small elementary building blocks connected by fixed bit permutations, where all the blocks are simple and can be of the same type. The resulting hardware designs are granular, simple, and fast, and can be customized easily by choosing different building blocks or connections among them. Consequently, the method is very suitable for data scrambling to thwart the probing attacks, as well as for new designs of hardware-oriented block ciphers. Moreover, the hardware implementations of such block ciphers can easily be made resistant to differential and simple power analysis attacks on the logical gate level by applying and adapting the masking technique [10] to the building blocks used. 
More details on data scrambling techniques together with some security requirements and considerations are given in Section 2. The DeKaRT method for designing key-dependent reversible transformations and logical circuits is proposed in Section 3 and some examples are presented in Section 4 The application of the DeKaRT method for the design of block ciphers is discussed in Section 5 and the application of the masking technique against power analysis is explained in Section 6. Conclusions are given in Section 7.

\section{$2 \quad$ Data Scrambling}

For an $n$-bit microprocessor, the typical block size to be dealt with by data scrambling is $n$ bits, but can be smaller. For example, apart from the data to be stored in RAM, the address of the location where the data has to be stored can be scrambled too. In addition, the scrambling function for data can be made dependent on the original address, for example, by deriving the key for this scrambling function from the same secret key and the original address by a simple hash function. Of course, scrambling the address foils the known plaintext scenario for scrambling the data, as the address of the location where the scrambled data is stored is not known. Not only is the address size usually smaller than $n$, but also need not be a power of 2 . Another example is scrambling the data and memory addresses within the microprocessor core (e.g., for the cache memory), where usually certain restrictions have to be respected when transmitting and storing the data. Accordingly, the block sizes as small as 8 bits or even smaller are likely to be encountered in data scrambling. For such block sizes, the key-controlled bit permutations cannot provide enough uncertainty of the key used.

For very small block sizes, data scrambling is inherently vulnerable to the dictionary attack, in the known or chosen plaintext scenario. This attack reconstructs the secret scrambling transformation used, and not the secret key itself. Secret key reconstruction attacks are more important because the same secret key bits can be used repeatedly for scrambling different data. However, it is important to have in mind that in the context of probing attacks, the known plaintext and known ciphertext scenarios are not realistic due to the fact that the ciphertext is likely to be known only partially.

If an iterated construction is used for data scrambling and if a number of secret key bits is incorporated in each of a small number of rounds (e.g., 2 to 5), then the effective key size is roughly halved due to the structural meet-in-themiddle attack in the known plaintext scenario. So, the number of key bits per round has to be relatively large, and this is not easy to achieve for very small block sizes. In particular, as proposed in [3], one can use a network composed of a small number of alternating substitution and key-controlled bit permutation layers, where the substitution layers consist of fixed and small (e.g., $(4 \times 4)$-bit) reversible S-boxes. For 5 layers altogether, because the bit permutations are linear functions for a given key, the structural attack 2] in the chosen plaintext scenario is then applicable, as already noted in [3]. The attack is able of recon- 
structing both the S-boxes, if they are unknown to the attacker, and the bit permutations in all the layers, up to an equivalence of the total transformation, and works even for large block sizes.

The secret key used for data scrambling should better be innovated for each new execution of the cryptographic function on the chip considered, having in mind that changing the key for scrambling the data in RAM can be done only when the stored data is all used. As a consequence, the secret key is much less exposed to side-channel attacks such as the power analysis attacks and hence the data scrambling schemes need not in principle be protected by the masking technique [9], 10] which randomizes (and slows down) the key-dependent computations. The secret key should be completely independent of the secret key stored on the chip which is used for the cryptographic function itself. It can be generated by a random number generator implemented in hardware on the same chip or, alternatively, but less securely, by a relatively strong and simple pseudorandom number generator (stream cipher), implemented in hardware, from a secret seed and some innovation information, which does not have to be secret. It is important to emphasize that the secret key used for scrambling should be stored in a hardware-protected register, not in RAM.

\section{DeKaRT Method}

Our strategic objective stemming from data scrambling applications described in previous sections is to propose a generic method for constructing key-dependent reversible transformations $\{0,1\}^{N} \rightarrow\{0,1\}^{N}$ by logical (combinatorial) circuits that can incorporate a relatively large number of key bits with a relatively small number of logical gates arranged in a small number of levels, even if the block size $N$ is very small, such as $N=8$.

In iterated constructions of block ciphers, where the block size $N$ is at least 64 , the number of key bits per each round is typically at most $N$ and the key bits are incorporated by using the bitwise XOR operation. Each round typically contains a layer of fixed nonlinear S-boxes, independent of the key, to provide confusion and may contain an extra layer of fixed affine transformations, also independent of the key, to provide diffusion. In a cipher like AES, the S-boxes have to be reversible, but act on all the input bits. If a Feistel structure is used, like in DES, then S-boxes do not have to be reversible, but act on a portion of input bits only. So, if $N$ is small, then a large number of rounds is needed to incorporate a large number of key bits, and this is not acceptable for data scrambling as the depth of the corresponding logical circuit would be too large.

For data scrambling purposes, the number of key bits per round can be increased by using key-controlled bit permutations to incorporate the key, as suggested in [3]. However, for small $N$ such as $N \leq 16$, this is not sufficient. Also, the key-controlled bit permutations are not cryptographically strong themselves. For example, regardless of the key, they preserve the Hamming weight of input data and, as a consequence, the XOR of all the output bits is always equal to 
the XOR of all the input bits. On the other hand, the computation of S-boxes does not involve any key and takes a considerable number of logical levels.

Accordingly, what we essentially need is an iterated construction composed of a number (not too large) of layers, where each layer implements a key-dependent reversible transformation, has a small logical depth, and is able to incorporate a number of key bits larger than the block size. A small logical depth in fact implies that each output bit of each layer depends on a small number of input bits to that layer. In the construction that we propose each layer consists of a number of small building blocks, each block implementing a key-dependent reversible transformation.

A generic bulding block is shown in Fig. 1 It acts on a small number of input data bits which are divided into two groups of $m$ and $n$ bits, respectively. The $m$ input bits are used for control and are passed to the output intact, like in the Feistel structure. They are then used to select $k$ out of $2^{m} k$ key bits by the multiplexer (MUX) circuit with $m$ control bits, $2^{m} k$-bit inputs, and the $k$-bit output k. The MUX circuit in fact implements an $m \times k$ lookup table, i.e., $k$ (binary) $m \times 1$ lookup tables that are specified by the key. Finally, the $k$ bits are then used to select an $(n \times n)$-bit reversible transformation $R_{\mathbf{k}}$ acting on the remaining $n$ input bits to produce the corresponding $n$ output bits. The $m$ and $n$ bits are called the control and transformed data bits, respectively. The total number of the key bits in the building block is thus $k 2^{m}$, which can easily be made larger than $m+n$. The set of used reversible transformations has to be chosen in a way that can easily be implemented by a logical circuit with $n+k$ input bits and $n$ output bits. The inverse building block is the same except that the reversible transformations $R_{\mathbf{k}}$ are replaced by their inverses $R_{\mathbf{k}}^{-1}$.

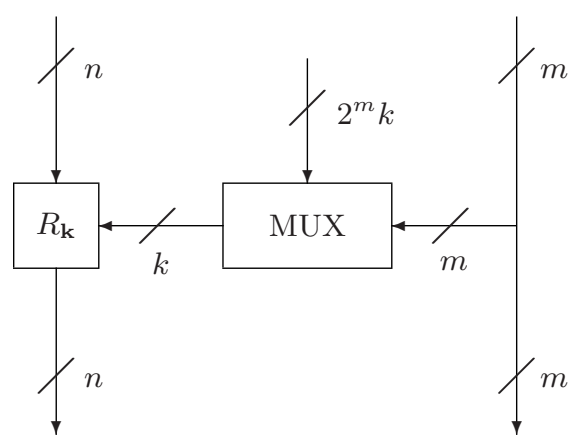

Fig. 1. The generic DeKaRT building block.

The underlying design paradigm is that a part of input data chooses a key and the key chooses a reversible transformation acting on the remaining part of input data. This justifies the name data-chooses-key-chooses-reversible_transformation 
and the notation $\mathrm{D} \rightarrow \mathrm{K} \rightarrow \mathrm{RT}$ for such a paradigm. For simplicity, we propose the name DeKaRT.

Consequently, in each layer, $N$ input bits are divided into small blocks and each of them is transformed by an elementary DeKaRT block. In a uniform design all the building blocks are of the same type. The layers are connected by fixed bit permutations satisfying the following two diffusion properties. In a uniform design, the bit permutations between the layers are the same. First, the control bits in each layer should be used as the transformed bits in the next layer. Therefore, the number of control bits in each layer cannot exceed the number of the transformed bits. In a uniform design, this implies that $m \leq n$. Second, for each building block, both control bits and transformed bits should be extracted from the maximal possible number of building blocks in the preceding layer. In a uniform design, this number equals $\min (m, N /(m+n))$ for the control bits and $\min (n, N /(m+n))$ for the transformed bits. In the inverse DeKaRT network, the layers are applied in the reverse order and the inverse bit permutations are used. If $m=n$, then the used bit permutations can be made equal to their inverses.

The definition given above is quite general, but is already sufficient for proposing specific constructions. Some concrete examples for the DeKaRT building blocks are given in the next section. For cryptographic security, a number of desirable additional criteria are also proposed.

- First, regarding the choice of $m+n$, it is prudent to require that the number of building blocks per each layer is at least 2 .

- Second, regarding the choice of the reversible transformations $R_{\mathrm{k}}$, it can be required that each output bit of $R_{\mathrm{k}}$ is a nonlinear function of input data bits and the key $\mathbf{k}$. Moreover, it can also be required that the algebraic normal form of this function contains at least one binary product involving both input data and key bits. In this way, the transformed and control input bits at each layer are nonlinearly combined together. This criterion implies that $n>1$, as the only reversible functions of one binary variable are the identity and the binary complement functions, so that the single key bit has to be XORed with the input bit to obtain the output bit. Already for $n=2$ this criterion can be satisfied, as shown in the next section. It is not satisfied if $k=n$ key bits are bitwise XORed with $n$ input data bits, as in the usual Feistel structure.

- Third, it is desirable that the set of reversible transformations $R_{\mathbf{k}}$ satisfies a Shannon-type criterion that the uncertainty of $n$ input bits provided by purely random $k$ key bits when the output $n$ bits are known is maximal possible, that is, $n$ bits. For this to hold, it is necessary that $k \geq n$. This criterion can easily be satisfied by bitwise XORing a subset of $n$ key bits with $n$ input data bits.

Cryptanalysis of the DeKaRT networks with a small number of rounds is a problem interesting for future investigations. To this end, the method from [4] developed for Feistel networks with four rounds and randomly chosen round functions may be relevant. 


\section{Examples}

In order to specify a concrete DeKaRT building block, one has to choose the parameters $m, n$, and $k$ and to propose a logical circuit to implement the parametrized reversible transformation $R_{\mathbf{k}}$ as a function of $n$ input data bits and $k$ key bits. This logical circuit should be relatively simple in terms of size and depth.

A simple and sufficient method for designing such logical circuits is to use XORs with 2 input bits and (controlled) SWITCHes only, where a SWITCH has 2 input bits, 2 output bits, and 1 control bit that determines if the input bits are swapped or not. Clearly, a SWITCH can be implemented by using two MUXes in parallel, whereas only one MUX suffices for implementing each XOR. Here and throughout, unless specified differently, a MUX has 2 input bits, 1 control bit, and 1 output bit. For each XOR, one of the two input bits is a key bit, whereas for each SWITCH, the control bit is a key bit. The individual key bits are incorporated into the circuit in such a way that there are no equivalent keys, i.e., that different combinations of key bits give rise to different reversible transformations. This is not a problem for checking since the parameters $n$ and $k$ are small. For each fixed key, such reversible transformations are affine, and the nonlinearity is achieved by the selected key bits depending on the control input data bits. Note that for $n=2$, all 24 reversible transformations of 2 input bits are necessarily affine. The Shannon-type criterion is not satisfied if the circuit contains the key-controlled SWITCHes only. The resulting DeKaRT building blocks thus incorporate, extend, and generalize, on an atomic level due to small block sizes, elements of known block cipher design principles such as Feistel structures [13, data-dependent bit permutations [14], 11], and key-dependent bit permutations 3 .

The basic concrete example satisfying the desirable properties from Section 3 with parameters $(m, n, k)=(2,2,3)$ is shown in Fig. 2 Many other examples can be obtained similarly. First, by removing the 2 XORs and 1 control input we get a DeKaRT block with parameters $(1,2,1)$, which will be called the simplified block from Fig. 2 Second, by removing 1 control input we get a block with parameters $(1,2,3)$. Third, by removing the SWITCH we get a block with parameters $(2,2,2)$. Fourth, a very elementary block with parameters $(1,1,1)$ contains only 1 MUX with 2 input key bits and 1 XOR for the reversible transformation. The DeKaRT block from Fig. 2 can be implemented by using a circuit of 13 MUXes with depth 4, whereas a circuit implementing the corresponding simplified block has size 3 and depth 2, also in terms of MUXes. The two blocks incoroporate the total of 12 and 2 key bits, respectively.

The blocks can readily be used for defining concrete data scrambling functions of the DeKaRT type. For example, for $N=16$, in the uniform DeKaRT network based on the block from Fig. 2, each layer contains 4 such blocks and hence has the total of 42 MUXes, has depth 4, and incorporates 48 key bits. Accordingly, five layers like this incorporate 240 key bits and can be implemented by a circuit with 210 MUXes and depth 20. Similarly, for $N=15$, in the uniform DeKaRT network based on the simplified block from Fig. 2, each layer contains 


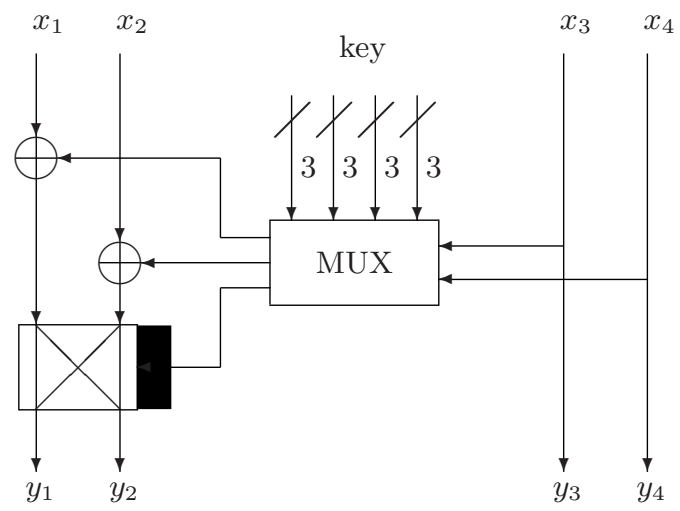

Fig. 2. An elementary DeKaRT building block.

5 such blocks and hence has the total of 15 MUXes, has depth 2, and incorporates 10 key bits. Accordingly, ten layers like this incorporate 100 key bits and can be implemented by a circuit with 150 MUXes and (the same) depth 20. It may be possible to further reduce the size and/or depth by using an optimized ASIC design, where the optimization also depends on the fixed bit permutations used between the layers. Both the networks incorporate a relatively large number of key bits and have a very small size and depth, which, for a relatively small $N$ such as $N \leq 16$, is impossible to achieve with the networks of S-boxes and key-controlled bit permutations. Also, due to the DeKaRT paradigm, their cryptographic security is considerably improved.

In conclusion, the proposed DeKaRT method is a new and interesting tool to be used for data scrambling purposes. Depending on the size and depth constraints stemming from particular applications, one can either use the proposed concrete DeKaRT designs or easily derive new customized concrete designs by using various DeKaRT building blocks as well as various bit permutations to be used between the layers.

\section{Application for Block Ciphers}

The generic or concrete DeKaRT designs described in the preceding section can also be used for constructing high-speed and/or small-size block ciphers suitable for hardware implementations. For example, they may be used for the (proprietary) encryption of copyright digital data to be stored in non-volatile memories for multimedia applications. What is needed is to use sufficiently many DeKaRT building blocks per layer according to the increased block size, $N \geq 64$, and to increase the number of layers/rounds to achieve a required security level, which is higher than for data scrambling applications. Namely, it is required that it should be computationally infeasible to reconstruct the secret key faster than by exhaustive search, given any number of arbitrarily chosen plaintextciphertext pairs. Since the size and depth of each DeKaRT layer is considerably 
smaller than in a usual iterated construction of block ciphers, the number of rounds in the DeKaRT construction can be several times larger. For example, for the DeKaRT building block from Fig. 2 and $N=128$, the number of rounds can be about 32 or larger.

For cryptographic security, one should satisfy the desirable criteria from Section 3 as well as possibly introduce two more round keys of size $N$ to be bitwise XORed with the input and output bits. Furthermore, in view of the statistical cryptanalytic methods such as the linear cryptanalysis of block ciphers [8], instead of using only the bit permutations between the layers, it is prudent to use very simple reversible linear functions. For example, if the total numbers of transformed and control data bits per layer are equal, one can design the bit permutations as explained in Section 3 and then XOR every transformed data bit at the input to each layer with a distinct transformed data bit from the preceding layer. This usually does not increase the logical depth of the layers. For the DeKaRT building block from Fig. 2] a preliminary analysis shows that less than 32 rounds are then sufficient to achieve resistance to the linear cryptanalysis, provided that the round keys are purely random and independent.

Unlike the data scrambling functions, the encryption or decryption functions do not have to be performed in only one microprocessor clock cycle, so that they can be implemented by a combination of logical circuits and registers. For example, several layers at a time can be implemented by a logical circuit. Note that the pipelined architectures for the DeKaRT constructions are extremely fast due to the small depth of each layer.

For the DeKaRT design, the required number of key bits per round is typically larger than the block size. This is needed for data scrambling applications where the block size and the number or rounds are both relatively small. For example, the DeKaRT building block from Fig. 2 requires 3 key bits per input bit. Furthermore, as for block cipher applications the number of rounds is increased, the total number of key bits required is larger than in usual block cipher designs. These key bits can be produced from a smaller number of secret key bits, stored in RAM or in a hardware-protected register, by a key expansion algorithm.

The key expansion algorithm can produce the round keys iteratively and can itself be implemented in hardware by a combination of logical circuits and registers, so that not all the round keys have to be stored. The relations between the round keys should not facilitate the secret key reconstruction attacks in the chosen plaintext scenario and should prevent the secret key reconstruction attacks in the related key scenario. The proposed DeKaRT variant of the key expansion algorithm is as follows. Let $K$ and $K^{\prime}$ denote the bit sizes of the secret key and the round key, respectively. The $K$ secret key bits are first expanded by linear transformations into $K^{\prime}$ key bits by using an appropriate linear code so that any subset of $K^{\prime \prime}$ expanded key bits are linearly independent, where $K^{\prime \prime}$ is relatively large $\left(K^{\prime \prime} \leq K\right)$. In other words, the minimum distance of the dual of this linear code should be at least $K^{\prime \prime}+1$ (e.g., see [7]). The obtained expanded key is then used as an input to a DeKaRT network of block size $K^{\prime}$ which is parametrized by a fixed randomly generated key satisfying an additional 
condition that every MUX block in the network implements balanced binary lookup tables. The $K^{\prime}$ bits produced after every two layers of the DeKaRT network are successively used as round keys, together with the $K^{\prime}$ input bits. For the decryption transformation, the round keys can be produced in the reverse order starting from the round key for the last round, which can be precomputed and stored.

As the number of layers is thus doubled when compared with the DeKaRT network used for the block cipher, the DeKaRT building blocks used could be as simple as the simplified block from Fig. 2. Alternatively, the $K^{\prime}$ round key bits can be produced after each layer of the DeKaRT network, if one allows portions of successive round keys to be bit permutations of each other. In the iterated DeKaRT algorithm each round is a reversible transformation, which is important as it satisfies the criterion that each round key is purely random if the input to the first round is purely random.

The key expansion algorithm can be simplified by using only linear transformations in the following way. The $K$ secret key bits are first expanded by linear transformations into $2 K^{\prime}$ key bits by using an appropriate linear code so that there are no small subsets of linearly dependent expanded key bits. The expanded $2 K^{\prime}$ bits are then used as the round keys for the first two rounds, whereas the subsequent pairs of successive round keys are produced by applying fixed bit permutations to the expanded key bits. Of course, other simplifications or modifications are also possible.

\section{DeKaRT Construction with Masking against Power Analysis}

Side-channel attacks on software or hardware implementations of various cryptosystems aim at recovering the secret key information from certain physical measurements performed on the electronic device during the computation such as the power consumption, the time, and the electromagnetic radiation. They do not change the functionality of the device and are typically not invasive. Power analysis attacks [6] are very powerful as they do not require expensive resources and as most (software) implementations without specific countermeasures incorporated are vulnerable to such attacks. Among them, the (first-order) differential power analysis (DPA) attacks are particularly interesting, because they use a relatively simple statistical technique that is almost independent of the implementation of the cryptographic algorithm. More sophisticated statistical analysis of power consumption curves may also be feasible.

The basis of power analysis attacks on cryptographic electronic devices are elementary computations within the device that depend on the secret key information and possibly on the known output and input information. If in addition the power consumption corresponding to these elementary computations depends on the input data, then it is not surprising that the power consumption curves contain information about the secret key which may be feasible to extract by statistical techniques. Software implementations, in which the oper- 
ations are synchronized by the microprocessor clock, are especially vulnerable. Hardware implementations are also potentially vulnerable, but may require a higher sampling frequency for obtaining the power consumption curves. A general algorithmic strategy to counteract power analysis attacks is to randomize the computations depending on the secret key by masking the original data with random masks and by modifying the computations accordingly [9], 10]. An alternative way of dealing with power analysis attacks is making use of a special encoding of data that tends to balance the power consumption, such as the dual-rail encoding, and the corresponding self-checking logical circuits, possibly asynchronous (see [12]).

The DPA attack on a microelectronic device implementing a cryptographic algorithm can be prevented if every elementary computation involving the secret information and performed by a logical gate in the hardware implementation is randomized. More precisely, the general condition to be satisfied is that the output value of each logical gate in the protected hardware design should follow the same probability distribution for each fixed value of the secret key and input information, where the uncertainty is provided by purely random masks. In other words, it should be statistically independent of the secret key and input information. In principle, even only one logical gate violating the condition may render the hardware implementation vulnerable to DPA. It may be interesting to note that randomizing the software operations does not ensure that the underlying hardware operations are all randomized.

In principle, for a logical gate with $m$ binary inputs only $m$ independent masking bits are sufficient, but this number can possibly be reduced. Of course, the greater the total number of masking bits used in the whole circuit, the greater the resistance to more sophisticated power analysis attacks such as a higherorder DPA. So, the whole hardware implementation can be masked by masking individual logical gates, and masking a logical gate means finding an equivalent logical circuit that can securely compute the masked output from the masked inputs, where all the masks are binary and are XORed with the input and output bits. More precisely, for a logical gate implementing a Boolean function $f(X)$, the masked gate should implement the function $f^{\prime}\left(X^{\prime}, R, r\right)=f\left(X^{\prime} \oplus R\right) \oplus r$, where $R$ is a binary vectorial input mask and $r$ is a binary output mask. The computation is required to be secure if $X^{\prime}=X \oplus R$ and the computed output is then $f(X) \oplus r$, as desired. The masking bits should preferably be produced by a random number generator each time the cryptographic function is executed.

A general masking technique based on using the MUX gate with 2 input bits, 1 control bit, and 1 output bit is proposed in [10]. It essentially consists in representing a Boolean function by a tree of MUXes and then in masking the MUXes. The function values as binary constants are used as inputs to the top layer of MUXes in the tree and are all masked by the same masking bit, which is also the masking bit for the output. The main observation from [10] is essentially that a MUX as an elementary logical gate can be masked by using a cascade connection of a SWITCH and a MUX, where the SWITCH is controlled by the control masking bit and the MUX is controlled by the masked control bit. 
Namely, if the two input masking bits are the same, then this cascade produces the MUX output bit masked with the same mask as the two inputs. Moreover, the two outputs of the SWITCH are computed securely, that is, each of them is statistically independent of the original input bits. As a SWITCH can be implemented by a parallel connection of two MUXes, the masked MUX is thus securely implemented by using 3 MUXes and has a logical depth of 2 MUXes. Consequently, the resulting logical circuit for the masked Boolean function contains 3 times as many MUXes and has double depth in comparison with the original tree of MUXes. This is the price to pay for the protection against DPA. It is not specifically explained in [10] how to apply this technique to an arbitrary logical circuit.

An important issue which is not adressed in [10] is related to the atomicity of the MUX implementation in hardware. If the MUX output is produced in a single step by a single gate, then the masked MUX computation is secure. However, in practice the MUX is usually implemented by using the logical AND, OR, and NOT gates. Namely, if $c$ denotes the control input and $x$ and $y$ the two data inputs, then the MUX implements the Boolean function $\bar{c} \wedge x \vee c \wedge y$. Now, if in the masked MUX each of the 3 MUXes is implemented in this way, it can be proven that the output of each elementary gate used is computed securely.

As mentioned in Section 2, if a new secret key for data scrambling is produced for every new execution of the cryptographic function and since the constraints regarding the speed and size are very restrictive, then the DeKaRT networks used for data scrambling need not be protected against power analysis by a masking technique. On the other hand, if the DeKaRT network is used for a block cipher, it should better be protected by masking. All what is needed is to mask the individual DeKaRT building blocks used and this can be achieved by adapting the MUX-based masking technique [10] described above. It is interesting that the masked round key bits needed can themselves be securely computed by the masked DeKaRT network used for the key expansion algorithm. Note that the key expansion algorithm is not vulnerable to DPA as it does not involve any input data, but other power analysis techniques may be applicable. A DeKaRT building block can be masked by using a representation in terms of MUXes (or SWITCHes) and by replacing each MUX by a masked MUX. The masked SWITCH is equivalently a cascade connection of two SWITCHes being controlled by the control masking bit and the masked control bit, respectively. The only condition to be respected is that the two inputs for each MUX (or SWITCH) should be masked by the same binary mask and that the control bit should be masked by an independent binary mask.

The MUX block from Fig. 1 can directly be represented in terms of MUXes. In fact, it contains $k$ distinct trees of MUXes with a total of $k\left(2^{m}-1\right)$ elementary MUXes with 2 input bits, 1 control bit, and 1 output bit. In each of the $k$ trees there are $m$ levels of MUXes controlled by $m$ control data bits and $2^{m}$ key bits are used as inputs to the top level. Now, assume that $m$ control data bits are masked by independent masking bits as well as that $2^{m}$ key bits for each of the $k$ trees are masked by the same mask. Accordingly, if each MUX is replaced by 
a masked MUX, then each of produced $k$ output bits is masked by the same bit that is used for masking the $2^{m}$ key bits at the input to the top level of the corresponding tree and all the computations are secure. The $k$ masking bits can be independent, but other options are also possible. For example, they can all be the same, whereas all the masking bits for $m$ control data bits can also be the same. Note that these two masking bits have to be independent in order for the computation to be secure.

In order to mask the block implementing the key-dependent reversible transformations $R_{\mathbf{k}}$, the corresponding logical circuit should be represented in terms of MUXes. This is already the case with any logical circuit composed of XORs and SWITCHes, as suggested in Section 4, and in particular with the circuit shown in Fig. 2. Then masking can be achieved by replacing each SWITCH by a masked SWITCH and by keeping the XORs as they are, having in mind that the output mask for an XOR is the XOR of the two input masks. The constraints to be satisfied are that the two inputs for each SWITCH should be masked by the same binary mask and that the control bit should be masked by an independent mask as well as that the two inputs for each XOR should be masked by independent masks. If needed, the mask at any point can be changed by using an extra XOR with another independent mask.

In the particular example from Fig. 2, which can be taken as an elementary building block to produce iterated block ciphers, we have $(m, n, k)=(2,2,3)$. The MUX block containing 3 trees each composed of 3 MUXes can be masked as explained above. The logical circuit composed of 2 XORs and 1 SWITCH can be masked by keeping the XORs and by replacing the SWITCH by a masked SWITCH, i.e., by a cascade of two SWITCHes, while the masking bits should be assigned following the general guidelines given above. Namely, the main condition to satisfy is that the two input masks for the cascade of SWITCHes should be the same. This can be achieved without introducing two extra XORs to change the masks for the two data inputs $x_{1}$ and $x_{2}$ by using the following two assignments of masking bits.

Let $k_{1}, k_{2}$, and $k_{3}$ denote the key bits used for the 2 XORs and the SWITCH in Fig. 2 respectively. In the first assignment, the input data bits $x_{i}$ have independent masks $r_{i}, 1 \leq i \leq 4$. If $k_{1}$ and $k_{2}$ are masked by $r_{2}$ and $r_{1}$, respectively, then the two inputs to the masked SWITCH are masked by the same mask, $r_{1} \oplus r_{2}$, as desired. The third key bit $k_{3}$ can then be masked either by $r_{1}$ or $r_{2}$ and the output bits of the masked SWITCH are then both masked by $r_{1} \oplus r_{2}$. Two additional XORs at the output can be used to change this mask into $r_{1}$ and $r_{2}$ for $y_{1}$ and $y_{2}$, respectively. As a result, the output four masks are the same as the input four masks, but the masking bits for the round keys should be adapted to the masking bits for the data.

In the second assignment, the input data bits $x_{i}$ are all masked by the same mask $r$, whereas all the key bits are themselves also masked by the same mask $r_{0}$, which is independent of $r$. The two inputs to the masked SWITCH are then masked by the same mask, $r \oplus r_{0}$, and so are the two output bits $y_{1}$ and $y_{2}$. To maintain the same type of mask assignment, the mask for the inputs $x_{3}$ and $x_{4}$ 
can be changed into $r \oplus r_{0}$ by using two additional XORs. This does not increase the depth of the masked DeKaRT block which remains to be 7 MUXes. For the second assignment, it is easier to produce the masked round keys as their masks are independent of the masks used for the data. In particular, one can use only one masking bit for the whole data block and only one, independent masking bit for each round key. Then after each round, every intermediate ciphertext bit will be masked by the same masking bit and this bit changes from round to round depending on the round key masking bits. The masked round keys can be obtained analogously, by using the corresponding masked DeKaRT network.

\section{Conclusions}

The proposed DeKaRT method for constructing key-dependent reversible logical circuits is not only suitable for data scrambling functions, but can also be used for constructing a new general type of hardware-oriented block ciphers as well as the required key expansion algorithms. In addition, the resulting hardware designs can efficiently be protected against power analysis by a masking technique on the logical gate level.

Acknowledgment. The author is grateful to Renato Menicocci for pointing out the reference [10] as well as for fruitful discussions on the countermeasures against power analysis attacks.

\section{References}

1. R. Anderson and M. Kuhn, "Tamper resistance - a cautionary note," Proceedings of the 2. USENIX Workshop on Electronic Commerce, Oakland, California, pp. 1-11, Nov. 1996.

2. A. Biryukov and A. Shamir, "Structural cryptanalysis of SASAS," Advances in Cryptology - EUROCRYPT 2001, Lecture Notes in Computer Science, vol. 2045, pp. 394-405, 2001.

3. E. Brier, H. Handschuh, and C. Tymen, "Fast primitives for internal data scrambling in tamper resistant hardware," Cryptographic Hardware and Embedded Systems - CHES 2001, Lecture Notes in Computer Science, vol. 2162, pp. 16-27, 2001.

4. D. Coppersmith, "Luby-Rackoff: four rounds is not enough," Technical Report RC 20674, IBM, Dec. 1996.

5. J. Daemen and V. Rijmen, The Design of Rijndael: AES - The Advanced Encryption Standard. Berlin: Springer-Verlag, 2002.

6. P. Kocher, J. Jaffe, and B. Jun, "Differential power analysis," Advances in Cryptology - CRYPTO '99, Lecture Notes in Computer Science, vol. 1666, pp. 388-397, 1999.

7. F. J. MacWilliams and N. J. A. Sloane, The Theory of Error-Correcting Codes. Amsterdam: North-Holland, 1988.

8. M. Matsui, "Linear cryptanalysis method for DES cipher," Advances in Cryptology - EUROCRYPT '93, Lecture Notes in Computer Science, vol. 765, pp. 386-397, 1994. 
9. T. Messerges, "Securing the AES finalists against power analysis attacks," Fast Software Encryption - FSE 2000, Lecture Notes in Computer Science, vol. 1978, pp. 150-164, 2001.

10. T. Messerges, E. Dabbish, and L. Puhl, "Method and apparatus for preventing information leakage attacks on a microelectronic assembly," US patent No. US 6,295,606 B1, Sept. 25, 2001 (filed July 26, 1999).

11. A. A. Moldovyan and N. A. Moldovyan, "A cipher based on data-dependent permutations," Journal of Cryptology, vol. 15(1), pp. 61-72, 2002.

12. S. Moore, R. Anderson, R. Mullins, G. Taylor, and J. Fournier, "Balanced selfchecking asynchronous logic for smart card applications," Microprocessors and Microsystems, to appear.

13. National Bureau of Standards, "Data Encryption Standard," Federal Information Processing Standards Publication 46, Jan. 1977.

14. R. L. Rivest, "The RC5 encryption algorithm," Fast Software Encryption - FSE '94, Lecture Notes in Computer Science, vol. 1008, pp. 86-96, 1995. 